\title{
Importance of Local Variations in Antibiotic Consumption and Geographical Differences of Erythromycin and Penicillin Resistance in Streptococcus pneumoniae
}

\author{
César García-Rey, ${ }^{1 *}$ Lorenzo Aguilar, ${ }^{1}$ Fernando Baquero, ${ }^{2}$ Julio Casal, ${ }^{3}$ and Rafael Dal-Ré ${ }^{1}$ \\ Medical Department, GlaxoSmithKline, ${ }^{1}$ Clinical Microbiology Department, Hospital Ramón y Cajal, ${ }^{2}$ and \\ Microbiology Department, Centro Nacional de Microbiología, Instituto de Salud Carlos III, ${ }^{3}$ Madrid, Spain
}

Received 30 July 2001/Returned for modification 17 September 2001/Accepted 22 October 2001

\begin{abstract}
A geographical analysis of how commonly prescribed oral antibiotics are quantitatively and qualitatively responsible for the different local rates of erythromycin and penicillin resistance in Streptococcus pneumoniae in Spain is presented. From 1998 to 1999 a multicenter surveillance study yielded 1,684 consecutive $S$. pneumoniae isolates from community-acquired respiratory infections. Data on antibiotic sales in the retail market for the same period were gathered, and the corresponding defined doses per 1,000 inhabitants per day were calculated. Macrolides and $\beta$-lactams were considered separately. Macrolides were subdivided into thrice-, twice-, and once-a-day macrolides, and $\beta$-lactams were split into aminopenicillins and cephalosporins. Univariate Spearman nonparametric coefficients $(R)$ were calculated, and variables proving to be significantly associated $(P<0.1)$ were entered into several multiple lineal regression models. Ample variation in both resistance rates and antibiotic consumption was seen. Multivariate analyses showed that integrated consumption of both macrolides and $\beta$-lactams accounted well for erythromycin $\left(R^{2}=0.722 ; P=0.002\right)$ and penicillin $\left(R^{2}=0.706 ; P=0.002\right)$ resistance. Macrolides were more important drivers for local differences in both erythromycin and penicillin resistance than $\beta$-lactams were. Consumption of once-a-day macrolides was key for local erythromycin resistance variations. Cephalosporins were slightly more important penicillin resistance drivers than aminopenicillins were.
\end{abstract}

The increasing development of resistance in Streptococcus pneumoniae not only to a single antibiotic but to many antibiotics at the same time (5) (multiresistant S. pneumoniae) is a striking example of the evolutionary adaptability of bacterial populations to antibiotic action. Antibiotics seem to have selected specific subpopulations resistant to the antibiotics frequently prescribed for common infections.

The development and subsequent spread of resistant pneumococci do not follow the same pace in different regions of the world. While in Northern European countries, rates of resistance to erythromycin and penicillin remain low, in Spain, France, Hong Kong and certain U.S. states, these rates may be above $50 \%$ (5). However, even in low-risk countries, a steady progression of resistance over recent years is being observed $(1,2,12)$.

Antibiotics are increasingly recognized as the leading force in this growth of resistance $(8,9,11,20)$. Nevertheless, studies performed so far to confirm this issue can only indirectly support the hypothesis of antibiotic consumption as the main driver for resistance differences. Even studies exploring the temporal coincidence between increased antibiotic consumption and resistance do not provide straightforward causal evidence. If the hypothesis is correct, then a positive correlation between antibiotic use in a given location and the corresponding prevalence of resistance should be expected. The aim of this study was to explore this hypothesis as well as to try to

\footnotetext{
* Corresponding author. Mailing address: GlaxoSmithKline Medical Department, c/ Severo Ochoa, 2, P. T. M. Tres Cantos, 28760-Madrid, Spain. Phone: 3491807 5738. Fax: 3491807 0596. E-mail: cesar.garcia -rey@gsk.com.
}

ascertain the specific weight that certain subgroups of antibiotics have on the prevalence of resistance to penicillin and erythromycin in S. pneumoniae.

\section{MATERIALS AND METHODS}

From November 1998 to October 1999 a nationwide multicenter surveillance study was carried out at 17 different hospitals in Spain (18). At each center, consecutive pneumococcal isolates from community-acquired respiratory tract infections (nasopharyngeal samples were excluded) were kept at $-70^{\circ} \mathrm{C}$. Once a month they were thawed, seeded onto an enriched transport medium, incubated overnight at 35 to $37^{\circ} \mathrm{C}$, and shipped to a central laboratory (Instituto Valenciano de Microbiología, Valencia, Spain). This single central laboratory undertook the confirmation of the initial identification and the susceptibility testing by a semiautomated microdilution method (Sensititre; Trek Diagnostics, Inc., Westlake, Ohio) using Mueller-Hinton broth supplemented with 3\% lysed horse blood according to the guidelines of the National Committee for Clinical Laboratory Standards (16). Overall, 1,684 S. pneumoniae isolates were available for analysis.

Data on antibiotic consumption for the same period in which the surveillance was run (a complete 365-day period) were gathered from International Marketing Services Ibérica S.A. (Madrid, Spain), which provided the total number of antibiotic wholesaler sales per presentation per year in the retail market. The consumption of each antibiotic was then calculated as defined daily doses (DDD) per 1,000 inhabitants per day (DID). Official demographic data of the Spanish population as of 1 January 1998 were obtained from the Spanish National Statistics Institute (Fig. 1).

In order to make the statistical analyses possible, and given the considerable diversity of the oral antibiotics currently marketed in Spain, only the two main families, $\beta$-lactams and macrolides, were considered. $\beta$-Lactams were in turn subdivided into aminopenicillins and cephalosporins (narrow-spectrum penicillins were excluded due to their low consumption), whereas macrolides were split into those administered three times a day (TID) (erythromycin and spiramycin), twice a day (BID) (clarithromycin, roxithromycin, mydecamycin, and josamycin), and once a day (OD) (azithromycin and dirithromycin).

The local prevalence of resistance was obtained by dividing the number of resistant isolates by the total number of strains collected at a given site, and their $90 \%$ confidence intervals ( $90 \%$ CIs) were calculated by the binomial method. 


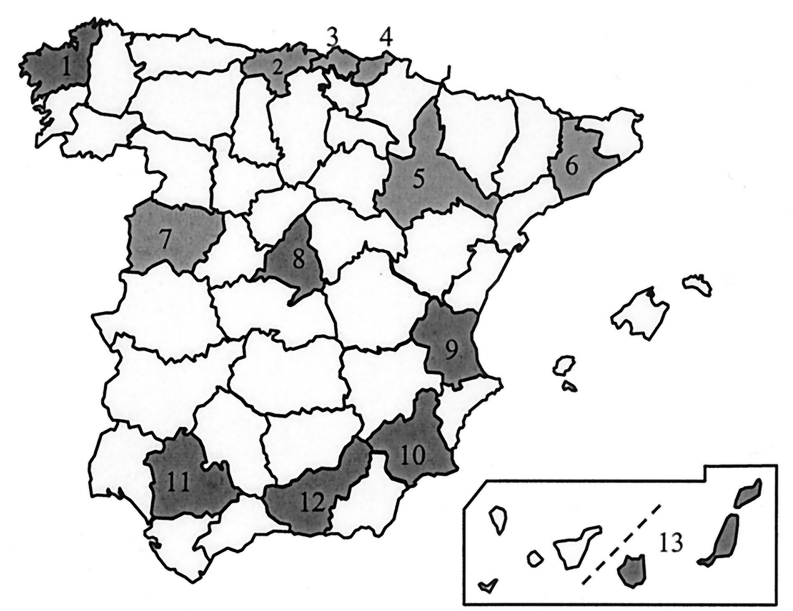

\begin{tabular}{|ll|}
\hline 1. La Coruña & (1,106,325 pop.) \\
2. Cantabria & (527,137 pop.) \\
3. Vizcaya & $(1,137,594$ pop. $)$ \\
4. Guipúzcoa & (676,439 pop.) \\
5. Zaragoza & (841,438 pop.) \\
6. Barcelona & $(4,666,271$ pop. $)$ \\
7. Salamanca & (349,550 pop.) \\
8. Madrid & (5,091,336 pop.) \\
9. Valencia & $(2,172,796$ pop. $)$ \\
10. Murcia & $(1,115,068$ pop. $)$ \\
11. Sevilla & $(1.714 .845$ pop. $)$ \\
12. Granada & $(801,177$ pop. $)$ \\
13. Las Palmas & (849,863 pop.) \\
\hline
\end{tabular}

FIG. 1. Sentinel provinces from which resistance and consumption data were drawn and their populations (pop.).

The composite results for two centers were used only for Madrid and Barcelona, which are highly populated. The remaining provinces had a single sentinel laboratory. To enter the analyses, provinces had to provide a minimum of 35 valid isolates. Penicillin ( $\mathrm{MIC} \geq 0.12 \mu \mathrm{g} / \mathrm{ml}$ ) and erythromycin ( $\mathrm{MIC} \geq 0.5 \mu \mathrm{g} / \mathrm{ml}$ ) were chosen as markers of resistance.

In order to ascertain the concurrent contribution that the antibiotic families and their subgroups might have on the resultant local differences in penicillin and erythromycin resistance, the prevalence of resistance in each province was plotted against each local antibiotic consumption value. For the macrolide models, erythromycin resistance was the dependent variable and provincial consumption values for global macrolides, TID, BID, and OD compounds, and $\beta$-lactams were the independent variables. The same procedure was followed for penicillin resistance and the consumption of oral $\beta$-lactams, aminopenicillins and cephalosporins, and macrolides.

Spearman nonparametric correlation coefficients $(R)$ with two-tailed significance to minimize the impact of outliers between provincial prevalence of resistance and antibiotic consumption were calculated in bivariate correlations. To integrate the effect of each of the independent variables that proved to be significantly associated $(P<0.1)$ in those correlations, multiple lineal regression modelling by the enter method was undertaken. The determination coefficient $\left(R^{2}\right)$ let us establish the goodness of the fitness of each model. Adjusted coefficients $(\beta)$ served to quantify the total influence of a given level of consumption on the prevalence of resistance (they can also be intuitively understood by looking at the figures), whereas nonadjusted coefficients $(B$, the coefficients of the regression equations) were a means to elucidate the same effect at equivalent
DIDs. The Statistical Package for Social Sciences for Windows, release 8.0, statistical package was used to carry out the analyses.

\section{RESULTS}

Significant geographical differences in erythromycin and penicillin resistance (Table 1 ) were clearly apparent. The lowest prevalences of erythromycin resistance were observed in Vizcaya $(22.2 \%$; $90 \%$ CI, 14.0 to 32.5$)$, Guipúzcoa $(27.5 \%$; $90 \%$ CI, 23.6 to 31.5 ), and Cantabria (29.8\%; $90 \%$ CI, 24.3 to $35.7)$, whereas the highest were observed in Murcia $(56.2 \%$; 90\% CI, 45.8 to 66.0), Zaragoza (47.5\%; 90\% CI, 36.5 to 58.7), and Valencia (44.2\%; $90 \%$ CI, 32.4 to 56.5 ).

Regarding penicillin resistance, the lowest prevalences occurred in Guipúzcoa (41.5\%; 90\% CI, 37.2 to 45.9$)$, Sevilla (42.8\%; $90 \%$ CI, 30.8 to 55.5$)$, and Vizcaya (44.4\%; $90 \%$ CI, 33.7 to 55.5$)$. The highest rates of penicillin resistance were seen in Zaragoza (68.8\%; 90\% CI, 57.7 to 78.5), Granada (66.6\%; $90 \%$ CI, 52.3 to 79), Salamanca (65.2\%; 90\% CI, 56.2 to 73.4$)$, and Murcia (64.4\%; $90 \%$ CI, 54.1 to 73.7$)$.

The quantitative contribution of pediatric samples for each

TABLE 1. Total number of isolates, prevalence of erythromycin and penicillin resistance, and antibiotic consumption by province

\begin{tabular}{|c|c|c|c|c|c|c|c|c|c|c|}
\hline \multirow{3}{*}{ Province } & \multirow{3}{*}{$\begin{array}{l}\text { Total } \\
\text { no. of } \\
\text { isolates }^{a}\end{array}$} & \multirow{3}{*}{$\begin{array}{l}\text { \% Showing erythromycin } \\
\text { resistance } \\
(90 \% \mathrm{CI})\end{array}$} & \multirow{3}{*}{$\begin{array}{l}\% \text { Showing penicillin } \\
\text { resistance } \\
(90 \% \mathrm{CI})\end{array}$} & \multicolumn{7}{|c|}{ Consumption (DDD/1,000 inhabitants/day) of: } \\
\hline & & & & \multicolumn{4}{|c|}{ Macrolides } & \multicolumn{3}{|c|}{$\beta$-Lactams } \\
\hline & & & & Total & TID & BID & OD & Total $^{b}$ & Aminopenicillins & Cephalosporins \\
\hline Granada & $39(15)$ & $41(27.7-55.4)$ & $66.6(52.3-73.4)$ & 3.38 & 1.02 & 1.83 & 0.52 & 16.30 & 11.83 & 3.82 \\
\hline $\begin{array}{l}\text { Sevilla } \\
\text { S }\end{array}$ & $49(6)$ & $32.6(21.6-45.2)$ & $42.8(30.8-55.5)$ & 2.58 & 0.91 & 1.21 & 0.46 & 14.12 & 9.95 & 3.28 \\
\hline Valencia & $52(7)$ & $44.2(32.4-56.5)$ & $50(37.9-62.1)$ & 4.34 & 1.12 & 2.49 & 0.73 & 16.76 & 11.69 & 4.75 \\
\hline Zaragoza & $61(2)$ & $47.5(36.5-58.7)$ & $68.8(57.7-78.5)$ & 4.00 & 1.35 & 1.86 & 0.80 & 15.32 & 11.23 & 3.51 \\
\hline Vizcaya & $63(13)$ & $22.2(14.0-32.5)$ & $44.4(33.7-55.5)$ & 2.80 & 0.81 & 1.38 & 0.61 & 13.78 & 10.02 & 3.20 \\
\hline La Coruña & $64(7)$ & $34.3(24.5-45.3)$ & $45.3(34.6-56.3)$ & 3.21 & 0.69 & 1.62 & 0.89 & 12.42 & 8.61 & 3.30 \\
\hline Murcia & 73 (12) & $56.1(45.8-66)$ & $64.4(54.1-73.7)$ & 3.91 & 1.01 & 2.04 & 0.86 & 18.25 & 12.93 & 4.64 \\
\hline Las Palmas & $82(0)$ & $42.7(33.4-52.3)$ & $51.2(41.6-60.7)$ & 4.05 & 1.29 & 2.00 & 0.76 & 15.74 & 10.94 & 3.6 \\
\hline Salamanca & $92(7)$ & $39.1(30.5-48.2)$ & $65.2(56.2-73.4)$ & 3.44 & 1.18 & 1.59 & 0.67 & 17.72 & 13.29 & 3.65 \\
\hline Cantabria & $188(43)$ & $29.8(24.3-35.7)$ & $48.9(42.7-55.1)$ & 2.60 & 0.67 & 1.48 & 0.45 & 14.65 & 11.59 & 2.47 \\
\hline Barcelona & $245(69)$ & $40.8(35.5-46.2)$ & $50.2(44.7-55.6)$ & 3.17 & 0.77 & 1.75 & 0.64 & 15.61 & 11.56 & 3.33 \\
\hline Madrid & $252(60)$ & $31.7(26.9-36.9)$ & $49.6(44.2-54.9)$ & 2.58 & 0.97 & 1.04 & 0.58 & 13.76 & 10.83 & 2.07 \\
\hline Guipúzcoa & $371(37)$ & $27.5(23.6-31.5)$ & $41.5(37.2-45.9)$ & 1.96 & 0.42 & 1.01 & 0.52 & 11.47 & 8.58 & 2.47 \\
\hline
\end{tabular}

${ }^{a}$ The number in parentheses is the number of pediatric isolates.

${ }^{b}$ Also includes narrow-spectrum penicillins. 

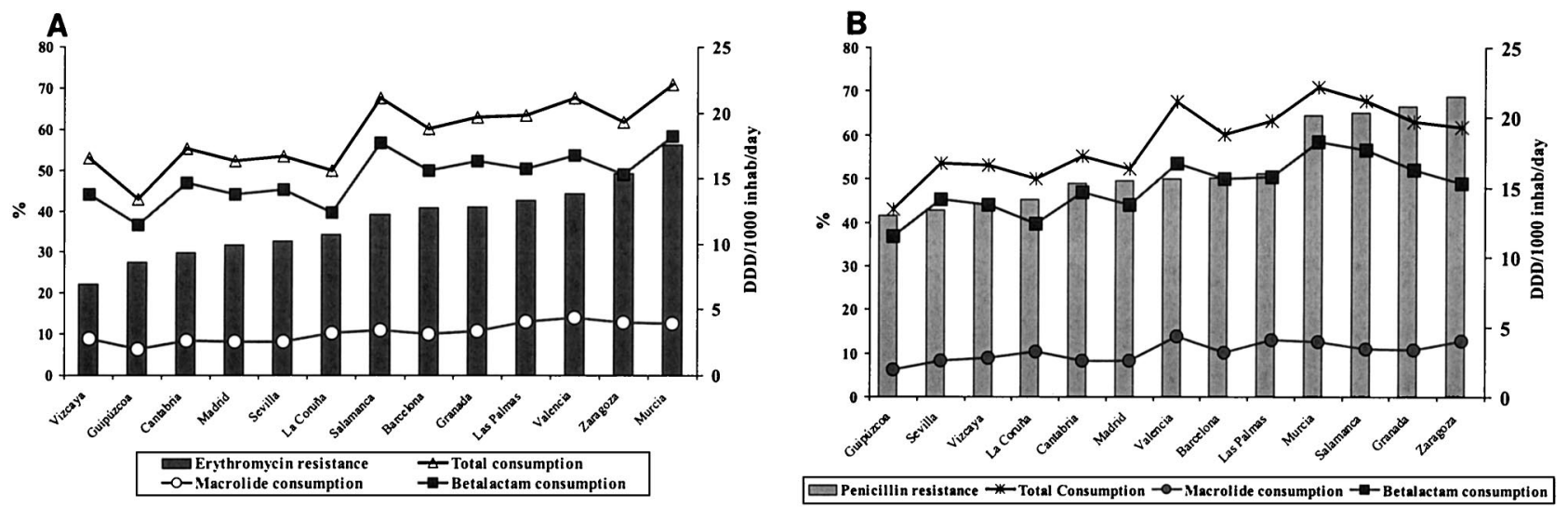

FIG. 2. Erythromycin (A) and penicillin (B) resistance and antibiotic consumption by province. inhab, inhabitants.

province, which ranged from 38.5 and $28.1 \%$ in Granada and Barcelona to 0 and $3.3 \%$ in Las Palmas and Zaragoza, is also shown in Table 1.

Local data on erythromycin and penicillin resistance, along with consumption of global macrolides, $\beta$-lactams, and their corresponding subgroups are also shown in Table 1 and are graphically depicted in Fig. 2 and 3. The whole study comprised several different univariate and multivariate analyses; results are shown in Table 2.

When looking at variations in provincial antibiotic consumption, sharp differences were again observed. Total macrolide consumption varied from a maximum of 4.34 DID in Valencia to a minimum of 1.96 DID in Guipúzcoa (ratio, 2.2). Qualitative differences were also identified, with a maximum TID macrolide use of 1.35 DID in Zaragoza and a minimum of 0.42 DID in Guipúzcoa (ratio, 3.2). A maximum BID macrolide consumption of 2.49 DID was shown in Valencia, opposed to a minimum of 1.01 DID in Guipúzcoa (ratio, 2.4). As for OD macrolide use, the highest consumption ( 0.89 DID) was seen in La Coruña and the lowest consumption ( 0.45 DID) was seen in Cantabria (ratio, 1.9).

Regarding the consumption of $\beta$-lactams, though quantitatively much more important than macrolide consumption, it presented a slightly narrower geographic fluctuation. The high- est value (18.25 DID) was obtained in Murcia and the lowest value (11.47 DID) was obtained in Guipúzcoa (ratio, 1.5). Aminopenicillin use was highest in Salamanca (13.24 DID) and lowest in La Coruña (8.59 DID) (ratio, 1.5), whereas cephalosporin consumption was highest in Valencia (4.56 DID) and lowest in Madrid (1.98 DID) (ratio, 2.3).

An association was seen between the geographical rates of erythromycin resistance and local consumption of macrolides $(R=0.850 ; P<0.001)$ and $\beta$-lactams $(R=0.758 ; P=0.003)$ (Fig. 2). The multiple regression model $1\left(R^{2}=0.722 ; P=\right.$ 0.002 ) showed that the specific weight of macrolides on the prevalence of erythromycin resistance was 1.7 times higher than that of $\beta$-lactam consumption ( $\beta$ value of 0.57 versus 0.34 , respectively) and increased nearly fivefold at equivalent consumption units ( $B$ values of 7.5 and 1.6, respectively).

For penicillin resistance, the bivariate correlations for macrolides and $\beta$-lactams were $0.864(P<0.001)$ and $0.780(P=$ $0.002)$, respectively. In multivariate model $2\left(R^{2}=0.706 ; P=\right.$ $0.002), \beta$ values of 0.49 and 0.41 reflected similar global contributions of macrolides and $\beta$-lactams to penicillin resistance, whereas at equivalent DIDs, the selection of penicillin resistance by macrolides was more than three times higher than by $\beta$-lactams ( $B$ values of 6.7 and 2 , respectively).

To assess and refine the accountability of the different anti-
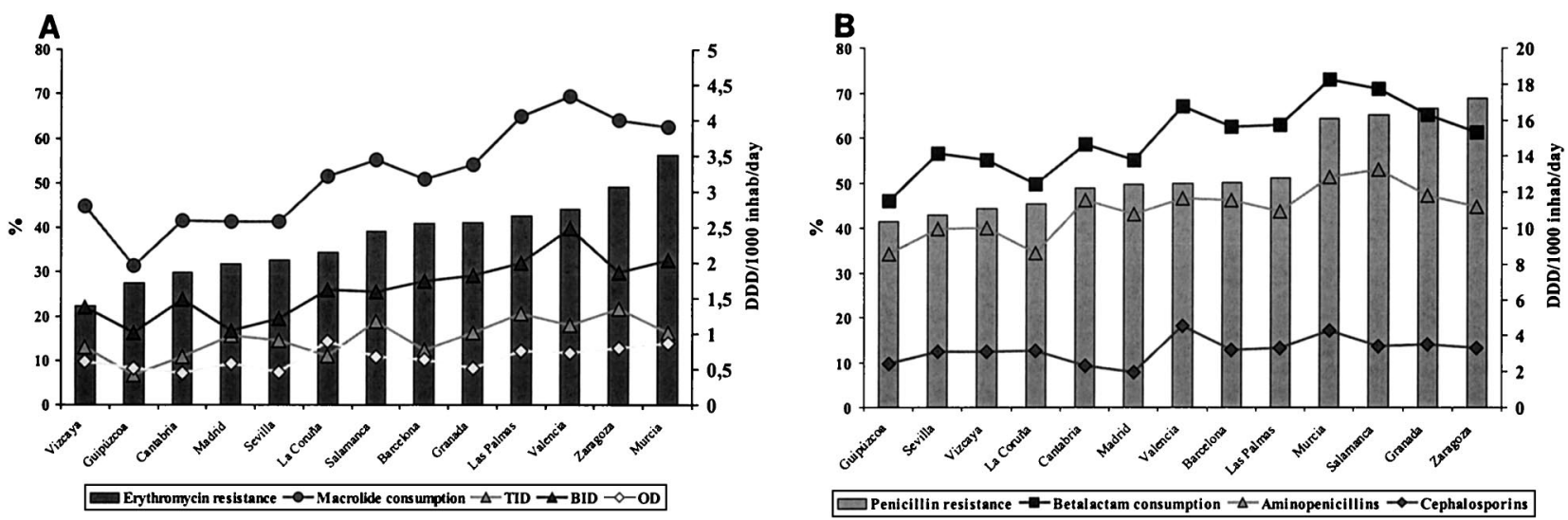

FIG. 3. (A) Erythromycin resistance and consumption of macrolides by province. (B) Penicillin resistance and consumption of betalactams by province. inhab, inhabitants. 
TABLE 2. Univariate and multivariate analyses for the four models tested

\begin{tabular}{|c|c|c|c|c|c|c|c|c|}
\hline \multirow{2}{*}{$\operatorname{Model}^{a}$} & \multirow{2}{*}{$\begin{array}{l}\text { Dependent } \\
\text { variable }\end{array}$} & \multirow{2}{*}{$\begin{array}{l}\text { Independent } \\
\text { variable }\end{array}$} & \multicolumn{2}{|c|}{$\begin{array}{l}\text { Result for univariate } \\
\text { analysis }\end{array}$} & \multicolumn{4}{|c|}{ Result for multivariate analysis } \\
\hline & & & $R$ & $P$ & $R^{2}$ & $P$ & Adjusted coefficient $\beta$ & Nonadjusted coefficient $B^{b}$ \\
\hline 1 & $Y_{E}$ & $\begin{array}{l}C_{M} \\
C_{\mathrm{BL}}\end{array}$ & $\begin{array}{l}0.850 \\
0.758\end{array}$ & $\begin{array}{r}<0.001 \\
0.003\end{array}$ & 0.722 & 0.002 & $\begin{array}{l}0.57 \\
0.34\end{array}$ & $\begin{array}{l}9.5 \\
1.6\end{array}$ \\
\hline 2 & $Y_{P}$ & $\begin{array}{l}C_{M} \\
C_{\mathrm{BL}}\end{array}$ & $\begin{array}{l}0.864 \\
0.780\end{array}$ & $\begin{array}{r}<0.001 \\
0.002\end{array}$ & 0.706 & 0.002 & $\begin{array}{l}0.49 \\
0.41\end{array}$ & $\begin{array}{l}6.7 \\
2.0\end{array}$ \\
\hline 3 & $Y_{E}$ & $\begin{array}{l}C_{\mathrm{TID}} \\
C_{\mathrm{BID}} \\
C_{\mathrm{OD}}\end{array}$ & $\begin{array}{l}0.725 \\
0.907 \\
0.646\end{array}$ & $\begin{array}{r}0.005 \\
<0.001 \\
0.017\end{array}$ & 0.674 & 0.014 & $\begin{array}{l}0.29 \\
0.44 \\
0.25\end{array}$ & $\begin{array}{r}10.0 \\
9.7 \\
15.8\end{array}$ \\
\hline 4 & $Y_{P}$ & $\begin{array}{l}C_{\mathrm{AMIN}} \\
C_{\mathrm{CEPH}}\end{array}$ & $\begin{array}{l}0.746 \\
0.731\end{array}$ & $\begin{array}{l}0.003 \\
0.005\end{array}$ & 0.646 & 0.006 & $\begin{array}{l}0.56 \\
0.37\end{array}$ & $\begin{array}{l}3.8 \\
5.0\end{array}$ \\
\hline
\end{tabular}

${ }^{a}$ Equations: model $1, Y_{E}=7.5 C_{M}+1.6 C_{\mathrm{BL}}-10.3 ;$ model $2, Y_{P}=6.7 C_{M}+2 C_{\mathrm{BL}}+0.8 ;$ model $3, Y_{E}=10 C_{\mathrm{TID}}+9.7 C_{\mathrm{BID}}+15.8 C_{\mathrm{OD}}+2.1 ;$ model $4, Y_{P}=$ $3.8 C_{\mathrm{AMIN}}+5 C_{\mathrm{CEPH}}-5.4$. Abbreviations: $Y_{E}$, erythromycin resistance; $Y_{P}$, penicillin resistance; $C_{M}$, macrolide consumption; $C_{\mathrm{BL}}, \beta$-lactam consumption; $C_{\mathrm{TID}}$, TID macrolide consumption; $C_{\mathrm{BID}}$, BID macrolide consumption; $C_{\mathrm{OD}}$, OD macrolide consumption; $C_{\mathrm{AmIN}}$, aminopenicillin consumption; $C_{\mathrm{CEPH}}$, cephalosporin consumption.

${ }^{b}$ Constants: model 1, -10.3 ; model 2, 0.8; model 3, 2.1; model 4, -5.4 .

biotic subgroups for their corresponding resistance markers, we employed two multivariate within-group models (Fig. 3). Univariate analysis for the macrolide model showed $R$ values of $0.725(P=0.005)$ for TID, $0.907(P<0.001)$ for BID, and $0.646(P=0.017)$ for OD macrolides. Multiple linear regression model 3 exploring the overall contribution of subgroups of macrolides to local erythromycin resistance $\left(R^{2}=0.674 ; P=\right.$ $0.014)$ showed that BID macrolides $(\beta=0.44)$ were twice as responsible as TID $(\beta=0.29)$ and OD $(\beta=0.25)$ macrolides for the differences observed. However, at equivalent consumption units (DID), OD macrolides $(B=15.8)$ were 1.5 times more likely than TID $(B=10)$ or BID $(B=9.7)$ macrolides to account for erythromycin resistance at a given site.

As far as penicillin resistance is concerned, univariate analyses provided $R$ values of $0.746(P=0.003)$ for oral aminopenicillin use and $0.731(P=0.005)$ for cephalosporin use. Multivariate model 4 also fit fairly well $\left(R^{2}=0.646 ; P=0.006\right)$. On this occasion, the $\beta$ coefficient for the consumption of aminopenicillins was 0.56 compared with 0.37 for cephalosporins (a 1.5 -fold increase in the global responsibility of aminopenicillins compared with that of cephalosporins). This finding was to be expected, as consumption of aminopenicillins was three to five times higher than that of cephalosporins. When adjusting for comparable levels of consumption, the local use of cephalosporins seems to be slightly more accountable for the prevalence of resistance to penicillin than does the use of aminopenicillins ( $B$ value of 5 versus 3.8, respectively).

\section{DISCUSSION}

Several factors have been proposed to explain the increase in penicillin and erythromycin resistance of $S$. pneumoniae that is occurring worldwide. These include clonal spread $(15,21)$, cyclic turnover of circulating serotypes $(13,19)$, natural fluctuations (22), different rates of mechanisms of resistance, mostly the $\mathrm{M}$ phenotype (efflux) in North America but the macrolide-lincosamide-streptogramin B phenotype in Europe (in the sample we used [18], 94.4\% of the erythromycin-resistant isolates showed the macrolide-lincosamide-streptogramin
B phenotype), and antibiotic consumption $(3,14)$. Several authors have taken on the task and have managed to show how resistance seems to closely follow antibiotic consumption over time. There are a number of studies that relate temporal variations in the consumption of antibiotics in the community to the development of resistance in key microorganisms $(9,11$, 20). In some instances, public health measures taken to halt the spread of resistance by diminishing antibiotic use have proved useful to a certain extent $(11,20)$.

If the assumption that antibiotic consumption is responsible for the spread of resistance is correct, then a correlation between local antibiotic use and a given level of resistance should be presumed. However, as penicillin and erythromycin resistance traits tend to associate in S. pneumoniae (7), the selection of resistance to a certain antibiotic marker by non-structurally related antibiotics would also be expected.

The data from the nationwide surveillance study SAUCE (18) offer a complete (in terms of the number of strains, methodology, and demographic considerations) and updated (from 1998 to 1999) view of the susceptibility of S. pneumoniae in Spain. As a result, the accuracy of the figures for the prevalence of resistance has been maximized. On the other hand, data on antibiotic consumption for the studied period are also very reliable. International Marketing Services Ibérica S.A. provides data on prescription sales (and even over-the-counter sales), not only in the public health system, but also in the private sector, thus covering up to $95 \%$ of the retail market. Nevertheless, it must be borne in mind that prescribing does not necessarily denote compliance, although it is thought to be an acceptable surrogate marker. The decision to use DDD per 1,000 inhabitants per day as a measure of antibiotic consumption was made so that results could be more easily compared among studies.

Two findings of this study are most striking: first, the variability in both erythromycin and penicillin susceptibilities exhibited by pneumococci isolated in different geographic areas of the same country, and second, the clear-cut differences in quantitative and qualitative levels of antibiotic consumption that cannot be explained by different levels of incidence of 
infection. It has been proposed that a critical threshold of 2 DID would be necessary for a cryptic resistance to be made apparent (10) and to trigger resistance. As current macrolide consumption is far above this hypothetical boundary, the present level of resistance to erythromycin in S. pneumoniae in Spain is not surprising.

The four multiple regression analyses performed seem to satisfactorily explain their outcome variables, with determination coefficients well above 0.6. Models 1 and 2 (for erythromycin and penicillin resistance, both with macrolide and $\beta$-lactam use as predictor variables) accounted for more than $70 \%$ of the variability of the dependent variables. For erythromycin and penicillin resistance, quantitative values were assigned to the contributions of macrolide and $\beta$-lactam use. Despite $\beta$-lactams being consumed much more than macrolides, this study strongly suggests that the overall responsibility for erythromycin resistance and even for penicillin resistance lies much more with the macrolides. Nonetheless, $\beta$-lactam use would be also responsible for a certain amount of the local prevalence of resistance to erythromycin.

However, as could be expected, taking into account the relative effects on resistance at equal levels of consumption, the analyses performed have also assigned to macrolides a more critical level of accountability than $\beta$-lactams for the differences observed in levels of erythromycin resistance and, astoundingly, in levels of penicillin resistance as well. In relative terms, and at equal levels of consumption, macrolides were almost fivefold more likely to select for erythromycin resistance, and threefold more likely to do so for penicillin, than were $\beta$-lactams. The bacteriostatic activity of macrolides, as opposed to the bactericidal $\beta$-lactam action, is a possible explanation.

In an attempt to further clarify responsibilities within these antibiotic groups, the behaviors of TID, BID, and OD macrolides on one hand and of aminopenicillins and cephalosporins on the other were ascertained. Logically, the $R^{2}$ values of these two models were somewhat smaller $(0.674$ and 0.646$)$. For macrolide model 3, as can be seen in Fig. 3A, BID macrolides were undoubtedly most responsible for the differences observed in resistance to erythromycin. However, on closer inspection and assuming equivalent consumption units (DID), OD macrolides $(B=15.8)$ were 1.5 times more prone than TID $(B=10)$ or BID $(B=9.7)$ macrolides to account for erythromycin resistance at a given site. As for penicillin model 4 , the global accountability of aminopenicillins was 1.5 times higher than that of cephalosporins, whereas at equal levels of consumption, the situation was reversed and cephalosporins were 1.3 -fold more likely to account for local penicillin resistance.

Taking the case of Murcia, with a consumption of 3.91 DID for macrolides and 18.25 DID for $\beta$-lactams, a predicted prevalence of erythromycin resistance of around $48 \%$ is obtained by applying model 1, compared to an observed value of $56.3 \%$. The predicted value was within the corresponding $90 \%$ CI for that province. If a reduction in consumption of macrolides (by 1.95 DID) to reach the level in Guipúzcoa was attained and the use of $\beta$-lactams remained constant, the resultant predicted prevalence of erythromycin resistance in Murcia would be $33 \%$, that is, a $31 \%$ decrease in the calculated value. A similar reduction of 1.95 DID in $\beta$-lactam consumption would attain an erythromycin resistance rate of $44.8 \%$ (a decrease of only $6.6 \%$ of the predicted value).

On the other hand, the predicted prevalence of penicillin resistance in model 2 for Zaragoza is $63.8 \%$ compared to the observed $68.8 \%$. If macrolide consumption decreased to reach that of Guipúzcoa (by 2.38 DID), with $\beta$-lactam use remaining constant, the expected penicillin resistance would be $48 \%$ (a $25 \%$ reduction). In order to get a similar reduction by modifying only $\beta$-lactam consumption and keeping macrolide consumption constant, it would be necessary to attain a much higher reduction of up to 9 DID, which would be extremely difficult. Therefore, small changes in the consumption of macrolides, rather than $\beta$-lactams, would be likely to have a much greater impact on penicillin resistance.

However, these striking results are not entirely unexpected. In vitro studies support a distinct potential among different macrolides (4) and $\beta$-lactams (17) to select for resistant strains. It seems that on a pharmacokinetic and pharmacodynamic basis, long-acting macrolides (with a low maximum concentration of drug in serum and a long half-life) may optimize selection of erythromycin resistance. Aminopenicillins seemed to be better selectors for low-level resistance than cephalosporins, and within cephalosporins, cefixime was the best resistance selector, probably due to the pharmacodynamic characteristics and selective antibiotic concentrations of any specific antibiotic (17). Likewise, clinical studies have described the seemingly higher capacity of macrolides than of $\beta$-lactams to select for resistant pneumococcal isolates among carriers (6). In this study, compared with the other antibiotic groups assessed, aminopenicillins seemed to have the lowest ecological impact on resistance, although they obviously have a measurable influence. Macrolides appeared to be the most important drivers of resistance to erythromycin and penicillin, and OD macrolides seemed to be more responsible for erythromycin resistance than TID and BID macrolides. It is highly probable that the differences found among TID, BID, and OD macrolides are in fact related to what these dosage schedules mean in terms of the relevant pharmacokinetic and pharmacodynamic parameters.

Some drawbacks must be acknowledged, the first being the relatively small sample size. The four multivariate analyses have only thirteen provincial observations, and therefore their statistical precision is not perfect. Nevertheless, this small sample size does not in any way affect the internal strength of the models, which is very high (very good explanatory usefulness), although it limits their predictive applicability.

The assumption that only antibiotic consumption accounts for resistance may also be criticized, since other potential or even not yet well-identified factors (i.e., clonal spread and clonal turnover) that may play a role have not been considered. However, while it is certain that such factors may be important, in the light of the high determination coefficients obtained (which point to a considerable external validity), they do not seem as crucial as antibiotic use. It would also have been worth knowing the antibiotic sales in previous years, since they might affect subsequent resistance. However, these figures were not available on a per gram basis. In addition, although trimethoprim-sulfamethoxazole use has been shown to be an important driving force for penicillin resistance in pneumo- 
cocci (7), this antibiotic was not considered due to its relatively low consumption in Spain.

Other criticisms may arise concerning the representativeness of the sampling, as detailed information on the populations may be sought to ensure the generalization of the results. However, health coverage in Spain is almost $100 \%$, and health resources are equitably distributed among the different regions in Spain. Of course, a selection bias might have occurred because the patients undergoing the specimen sampling may be more likely to have failed in previous treatments, because of different diagnostic activities in the provinces, or because of other special conditions. For example, the unequal distribution of pediatric isolates among the different survey sites (Table 1) might have led to overestimating or underestimating the calculated prevalence of resistance. The proportion of pediatric samples was higher in some sites possibly due to multiple factors, such as the real proportion of the child population and the logistics of sample processing in each area. In any event, this heterogeneity is likely to reflect the reality better than an ideal experiment can, since it is hardly feasible (and may not be advisable) to control every variable in population-based studies. Heterogeneity is unfortunately inherent in almost every surveillance study of this kind. However, while our results do not represent accurate reality (this is impossible to accomplish on a population basis), they can be considered good quality estimators of measurable reality, since all the consecutive pneumococcal isolates at each center were processed.

It must not be forgotten that this is an ecological study, and consequently, its conclusions by no means constitute a firm proof of cause. Causality can be obtained only under experimental conditions that are very unlikely to be complied with when dealing with human populations. Nonetheless, on a population level, the results of this study support the clinical and in vitro experimental observations of a distinct accountability of different antibiotics for a given rate of resistance.

To assess the dynamics of antibiotic resistance in a given country, analyses of a sufficiently high number of strains from an adequate number and distribution of sentinel laboratories, coupled with reliable data for antibiotic consumption, are needed. Similar studies would be desirable in other countries with different rates of resistance and antibiotic consumption, so as to refine this methodology to make it more useful for guiding tailored actions of antibiotic containment in an attempt to curtail the spread of resistance.

\section{ACKNOWLEDGMENTS}

We are grateful to A. Pedromingo and J. J. Granizo (Fundación Jiménez Díaz) for critical review of the statistical methodology, to each of the members of the Spanish Surveillance Group for Respiratory Pathogens for continued effort in updating the resistance patterns in Spain, and to Veronica A. Maguire for assistance with the English version of the manuscript.

\section{REFERENCES}

1. Baquero, F., and the Task Force of the General Direction for Health Planning of the Spanish Ministry of Health. 1996. Antibiotic resistance in Spain: what can be done? Clin. Infect. Dis. 23:819-823.

2. Baquero, F. 1995. Pneumococcal resistance to $\beta$-lactam antibiotics: a global perspective overview. Microb. Drug Resist. 1:115-120.
3. Baquero, F. 1999. Evolving resistance patterns of Streptococcus pneumoniae: a link with long-acting macrolide consumption? J. Chemother. 11(Suppl. 1):35-43.

4. Drusano, G. L., and W. A. Craig. 1997. Relevance of pharmacokinetics and pharmacodynamics in the selection of antibiotics for respiratory tract infections. J. Chemother. 9(Suppl. 3):38-44.

5. Felmingham, D., and R. N. Grüneberg. 2000. The Alexander Project 19961997: latest susceptibility data from this international study of bacterial pathogens from community-acquired lower respiratory tract infections. J. Antimicrob. Chemother. 45:191-203.

6. Ghaffar, F., I. R. Friedland, and H. McCracken. 1999. Dynamics of nasopharyngeal colonization by Streptococcus pneumoniae. Pediatr. Infect. Dis. J. 18:638-646.

7. Goldstein, F. W. 1999. Penicillin-resistant Streptococcus pneumoniae: selection by both $\beta$-lactam and non- $\beta$-lactam antibiotics. J. Antimicrob. Chemother. 44:141-144.

8. Granizo, J. J., L. Aguilar, J. Casal, R. Dal-Ré, and F. Baquero. 2000. Streptococcus pyogenes resistance to erythromycin in relation to macrolide consumption in Spain (1986-1997). J. Antimicrob. Chemother. 46:959-964.

9. Granizo, J. J., L. Aguilar, J. Casal, C. García-Rey, R. Dal-Ré, and F. Baquero. 2000. Streptococcus pneumoniae resistance to erythromycin and penicillin in relation to macrolide and $\beta$-lactam consumption in Spain (19791997). J. Antimicrob. Chemother. 46:767-773.

10. Huovinen, P., H. Seppälä, J. Kataja, T. Klaukka, and the Finnish Study Group of Antimicrobial Resistance. 1997. The relationship between erythromycin consumption and resistance in Finland. Ciba Found. Symp. 207:3646.

11. Kristinsson, K. G. 1997. Effect of antimicrobial use and other risk factors on antimicrobial resistance in pneumococci. Microb. Drug Resist. 3:117-123.

12. Liñares, J., F. Tubau, and M. A. Domínguez. 2000. Antibiotic resistance in Streptococcus pneumoniae in Spain: an overview in the 1990s, p. 399-407. In A. Tomasz (ed.), Streptococcus pneumoniae. Molecular biology and mechanisms of disease-update for the 1990s. Mary Ann Liebert, Inc., New York, N.Y.

13. Marco, F., E. Bouza, J. García-de-Lomas, L. Aguilar, and the Spanish Surveillance Group for Respiratory Pathogens. 2000. Streptococcus pneumoniae in community-acquired respiratory tract infections in Spain: the impact of serotype and geographical, seasonal and clinical factors on its susceptibility to the most commonly prescribed antibiotics. J. Antimicrob. Chemother. 46:557-564.

14. Melander, E., K. Ekdahl, G. Jönsson, and S. Mölstad. 2000. Frequency of penicillin-resistant pneumococci in children is correlated to community utilization of antibiotics. Pediatr. Infect. Dis. J. 19:1172-1177.

15. Muñoz, R., T. J. Coffey, M. Daniels, C. G. Dowson, G. Laible, J. Casal, R. Hakenbeck, M. Jacob, J. M. Musser, B. G. Spratt, and A. Tomasz. 1991. Intercontinental spread of a multiresistant clone of serotype 23F Streptococcus pneumoniae. J. Infect. Dis. 164:302-306.

16. National Committee for Clinical Laboratory Standards. 1997. Methods for dilution antimicrobial susceptibility tests for bacteria that grow aerobically, 4th ed. Approved standard M7-A4. National Committee for Clinical Laboratory Standards, Wayne, Pa.

17. Negri, M. C., M. I. Morosini, E. Loza, and F. Baquero. 1994. In vitro selective antibiotic concentrations of $\beta$-lactams for penicillin-resistant Streptococcus pneumoniae populations. Antimicrob. Agents Chemother. 38:122125.

18. Pérez-Trallero, E., C. Fernández-Mazarrasa, C. García-Rey, E. Bouza, L. Aguilar, J. García-de-Lomas, F. Baquero, and the Spanish Surveillance Group for Respiratory Pathogens. 2001. Antimicrobial susceptibilities of 1,684 Streptococcus pneumoniae and 2,039 Streptococcus pyogenes isolates and their ecological relationships: results of a 1-year (1998-1999) multicenter surveillance study in Spain. Antimicrob. Agents Chemother. 45:33343340.

19. Raymond, J., I. Le Thomas, F. Moulin, A. Commeau, D. Gendrel, and P. Berche. 2000. Sequential colonization by Streptococcus pneumoniae of healthy children living in an orphanage. J. Infect. Dis. 181:1983-1988.

20. Seppälä, H., T. Klaukka, J. Vuopio-Varkila, A. Muotiala, H. Helenius, K. Lager, et al. 1997. The effect of changes in the consumption of macrolide antibiotics on erythromycin resistance in group A streptococci in Finland. Finnish Study Group for Antimicrobial Resistance. N. Engl. J. Med. 337: 441-446.

21. Soares, S., K. G. Kristinsson, J. M. Musser, and A. Tomasz. 1993. Evidence for the introduction of a multiresistant clone of serotype 6B Streptococcus pneumoniae from Spain to Iceland in the late 1980s. J. Infect. Dis. 168:158163.

22. Stingemore, N., G. R. J. Francis, M. Toohey, D. B. McGechie, and J. Turnidge. 1990. The emergence of erythromycin resistance in Streptococcus pyogenes in Fremantle, Western Australia. Med. J. Aust. 150:621-631. 